\begin{abstract}
S *
Burke, Bernard F. Systematic distortion of the outer regions of the galaxy.

The distribution of hydrogen in the galaxy can be deduced from $2 \mathrm{I}-\mathrm{cm} H$-line data, using

\begin{tabular}{|c|c|c|}
\hline Object & $m_{\mathrm{pg}}$ & $m_{\mathrm{pv}}$ \\
\hline IC 2448 & $14.20 \pm 0.05$ & $14.50 \pm 0.30$ \\
\hline NGC 2792 & $15.90 \pm 0.20$ & $16.00 \pm 0.50$ \\
\hline NGC 2818 & $17.45 \pm 0.30$ & \\
\hline NGC 3132 & $10.62 \pm 0.20$ & $9.40 \pm 0.15$ \\
\hline I I $36^{\mathrm{m}},-5^{2^{\circ}} 39^{\prime}$ & I3.59 \pm 0.05 & \\
\hline
\end{tabular}
\end{abstract}
the Oort model. The Leiden and Australian groups have shown in unpublished work that if the Lund pole is shifted, the major concentration of hydrogen remains close to the galactic plane, with small deviations. The $2 \mathrm{I}-\mathrm{cm}$ data of both the Carnegie Institution and Leiden Observatories have been analyzed with respect to the new galactic plane derived by the Leiden and Australian groups, to see if the outermost. hydrogen in the galaxy showed deviations which could be attributed to tidal distortion by the Large Magellanic Cloud. A deviation does exist, the center of mass of the outermost hydrogen lying consistently below the plane on the side closest to the LMC, while the outermost hydrogen on the far side lies consistently above the plane. This deviation is as great as $300 \mathrm{pc}$ for principal condensations and even more for the tenuous outer edges. An order-of-magnitude calculation indicates that gravitational forces alone would not cause a deviation of more than about $30 \mathrm{pc}$ even for masses of $7 \times 10^{10}$ and $4 \times 10^{9}$ solar masses for the galaxy and LMC, respectively. The distortion, while in the proper direction, would thus appear to be considerably greater than one would expect from gravitational forces only. We understand that this same effect has independently been noted by J. F. Kerr and J. V. Hindman in Australia.

Department of Terrestrial Magnetism, Carnegie Institution of Washington, Washington, D. C.

\section{Camp, D. C. Magnitudes of central stars in five southern planetary nebulae.}

Magnitudes were obtained from fifty-three photographs taken at the request of Dr. Lawrence $\mathrm{H}$. Aller by Dr. Paraskevopoulos with the Harvard 6o-inch telescope at Bloemfontein, South Africa, during March, April, and May, I 948. The Harvard standard E regions were used for calibration. Descriptions and dimensions are given for the planetaries, with adopted photographic and photovisual magnitudes for five of the central stars being:
The relatively large probable errors result from the fact that so few plates were available.

\section{Goethe Link Observatory, Indiana University,} Bloomington, Ind.

\section{Coates, Robert J. Solar radiation at $4 \mathrm{~mm}$ wave length.}

Measurements of solar radiation at $4 \mathrm{~mm}$ wave length were started at the U. S. Naval Research Laboratory in July 1956 . The antenna used is a Io-foot precision parabolic reflector having a beam width of $6^{\prime}$. Scans across the disc of the sun have been made under different sunspot conditions over a period of several months. These indicate that the sun at $4 \mathrm{~mm}$ wave length is very close to a uniform disc with a diameter about one per cent larger than that of the optical disc. A slight increase of the order of two per cent is noted at the positions of large sunspots. The average of these solar measurements gives a corrected solar antenna temperature of $5600^{\circ} \mathrm{K}$ with an uncertainty of $\pm 400^{\circ} \mathrm{K}$. These measurements were corrected for the atmospheric attenuation which usually is the order of fifty per cent at the sun's altitude on the meridian.

Naval Research Laboratory, Washington, D. C.

\section{Cox, John P. Non-adiabatic stellar pulsation.}

The $V$-equation of Woltjer (1936), which determines the non-adiabaticity, and hence the flux variations, associated with stellar pulsation, has been solved in a first-order theory under quite general conditions along the lines developed by M. Schwarzschild (1938). The manner of mechanical pulsation of the star here is that given by the solution of the adiabatic wave equation. In particular, the flux variation at the

* Of papers presented at the Ninety-sixth Meeting of the American Astronomical Society, New York, N. Y., December 26-29, I956. 